\title{
Solvability of a dynamic rational contact with limited interpenetration for viscoelastic plates
}

\author{
Jiř́ Jarušek*
}

\begin{abstract}
The solvability of the rational contact with limited interpenetration of different kind of viscolastic plates is proved. The biharmonic plates, von Kármán plates, Reissner-Mindlin plates and full von Kármán systems are treated. The viscoelasticity can have the classical ("short memory") form or the form of a certain singular memory. For all models some convergence of the solutions to the solutions of the Signorini contact is proved provided the thickness of the interpenetration tends to zero.
\end{abstract}

Key words. Dynamic contact problem, limited interpenetration, viscoelastic plate, existence of solutions.

Mathematics Subject Classification. 35Q74, 74D10, 74H20, 74K20, 74M15.

\section{Introduction and notation}

Despite a great amount of actual and/or possible applications, the theory of contact problems remains still underdeveloped. The study of contact problems has been started by A. Signorini [12], [13]. His model describing a contact of a deformable body with a rigid foundation respects the impenetrability of Mass. It was extended to dynamic problems by L. Amerio, G. Prouse, M. Schatzman and further authors in late seventies and early eighties of the last century. The monograph [6] summed up the development in this field till its publication. The highly nonlinear Signorini model is complex. Therefore a bit later so called normal compliance approach has been introduced. This approach is nothing else than replacement of the original Signorini contact model by some kind of its penalization. Although such kind of approximation is a suitable auxiliary tool in the numerical investigation of contact problems, this approach has brought no deep results to their theory. It is usually easy to derive properties of solutions of such approximate problems and the real hard work starts by the limit process to the original problem.

However the normal compliance approach has drawn the attention to the fact that the complete impenetrability of Mass need not be completely physically realistic, because from the microscopical point of view no material is flat or smooth enough. Just in the medium advanced microscopes the seemingly perfectly flat or smoothly curved surfaces are seen as a huge collections of asperities and small holes or cavities. The asperities may be deformed or may fill the holes of the counterpart partially or completely. Hence it has some good sense to study models, where some interpenetration between body and the foundation is allowed to describe macroscopically those phenomena. However, to remain physically realistic, this interpenetration model must include a certain bound after which the further penetration is not possible. And, as well, it is realistic to assume that such a bound cannot be reached.

*Institute of Mathematics, Czech Academy of Sciences 
These are the premises of the rational contact model which was introduced by [7] and [8], where the solvability of its static version has been proved. The first dynamic (frictionless) rational contact has been investigated in [9]. It concerns a boundary contact of a body with a foundation.

Since 2006 a series of papers about the solvability of dynamic Signorini contact problems for different models of plates [1]-[4] was published. The purpose of this paper is to extend these results to the rational contact with limited interpenetration. Unlike [9] we face here a domain contact.

\section{Abstract formulation of the problem for the clamped or simply supported viscoelastic plate and the scheme of its solution}

Let $\Omega \subset \mathbb{R}^{2}$ be a bounded domain with a sufficiently smooth boundary $\Gamma$. Let $X$ be a Sobolev-type Hilbert space defined on $\Omega$, let $Y$ be the space of traces of elements from $X$ on $\Gamma$. Let $A, B: X \rightarrow X^{*}$ be two linear symmetric strongly elliptic operators in the form $\mathscr{D}^{*} a \mathscr{D}$, $\mathscr{D}^{*} b \mathscr{D}$, respectively, where $\mathscr{D}$ is a differential operator and $a, b$ are positively definite matrices or tensors of time constant but possibly space-dependent elements. Let $I \equiv[0, T]$ be a time interval. Here the dual space $X^{*}$ is defined via the suitable generalization of the $L_{2}(\Omega)$ scalar product. Let $\mathscr{X} \equiv L_{2}(I ; X)$. We introduce the bilinear forms $\mathscr{A}:\{u, v\} \mapsto\langle a \mathscr{D} u, \mathscr{D} v\rangle_{Q}$, $\mathscr{B}:\{u, v\} \mapsto\langle b \mathscr{D} u, \mathscr{D} v\rangle_{Q}$, where $\langle\cdot, \cdot\rangle_{Q}$ is the $L_{2}(Q)$ scalar product and $Q \equiv I \times \Omega$.. Let $S \equiv I \times \Gamma$ Let $E(t): X \rightarrow X^{*}$ be anoother operator.

We shall denote the elements of $v \in X$ or $v: I \rightarrow X$ such that $v \in \mathscr{X}$ as displacements, and their first time derivatives (denoted by dots) as velocities. Let $\gamma$ be a negative real number. Let $p: \mathbb{R} \rightarrow \overline{\mathbb{R}} \equiv \mathbb{R} \cup\{+\infty\}$ be a nonincreasing function such that $p(x)=0$ for $x \geq 0, p(x) \in \mathbb{R}$ for $x>\gamma$, and $\lim _{x \searrow \gamma} p(x)=+\infty$, where $\gamma \in \mathbb{R}$ is a given bound of the interpenetration. Our problem is to find $u \in \mathscr{X}$ such that $\dot{u} \in \mathscr{X}$ for which the following set of relations holds

$$
\begin{aligned}
\ddot{u} & =A \dot{u}+B u-E u+p(u+g)+f \text { in } X \text { on } I, \\
D(u) & =0 \in Y, \\
u(0) & =u_{0}, \dot{u}(0)=u_{1}
\end{aligned}
$$

Here $D$ is a general differential operator of a Dirichlet or somewhat combined type. If $X=$ $H^{2}(\Omega)$, the space of square integrable functions having the (possibly generalized) first and the second derivatives square integrable as well and $A, B$ are differential operators of the fourth order then $D(u) \equiv\left\{D_{1}(u), D_{2}(u)\right\}, D_{1}(u)=u-u_{0}$ for both cases, $D_{2}(u)=\partial_{\tilde{n}}\left(u-u_{0}\right)$ (the outer co-)normal derivative) or $D_{2}(u)=M(u)$ a Neumann-type operator, which ensures that after the integration by parts in the space variable in the variational formulation of the problem no additional boundary term occurs. The first couple describes a clamped plate while the second one a simply supported plate. Let us mention that $p(u+g)$ stands there for the contact force, where $g \geq 0$ is the gap function,

We shall define a sequence of auxiliary approximate problems to (1) by adding the following additional assumption on $p$ : We assume the existence of a sequence $\left\{\delta_{k}\right\} \subset \mathbb{R}_{+}$such that $\delta_{k} \searrow 0$ and for each $k \in \mathbb{N}$ there is a left derivative $\partial^{l} p$ in the points $\gamma+\delta_{k}, k \in \mathbb{N}$ such that $\partial^{l} p\left(\gamma+\delta_{k}\right) \geq \partial^{l} p\left(\gamma+\delta_{k+1}\right), k \in \mathbb{N}$ and $\lim _{k \rightarrow+\infty} \partial^{l} p\left(\gamma+\delta_{k}\right)=-\infty$. Then we define $p_{k}: y \mapsto \min \left\{p, p\left(\gamma+\delta_{k}\right)+\partial^{l} p\left(\gamma+\delta_{k}\right)\left(y-\gamma-\delta_{k}\right)\right\}$ for $y \leq \gamma+\delta_{k}, p_{k}=p$ elsewhere and the auxiliary problem is defined by replacement of $p$ by $p_{k}$ in (11). 
Let us denote by $\langle\cdot, \cdot\rangle_{\Omega}$ the duality pairing of $X$ and $X^{*}$ derived from the $L_{2}(\Omega)$ scalar product and by $\langle\cdot, \cdot\rangle_{Q}$ the duality pairing of $\mathscr{X}$ and $\mathscr{X}^{*}$ derived from the $L_{2}(Q)$ scalar product. Let $\mathscr{X}_{0}$ be a subspace of elements of $\mathscr{X}$ satisfying the appropriate homogeneous Dirichlet boundary condition in (1), let $\mathscr{X}_{1} \equiv\left\{v \in \mathscr{X}_{0} ; \dot{v} \in L_{2}(Q)\right\}$.

Multiplying the first row of (1) by a test function $v \in \mathscr{X}_{0}$ and performing the integration by parts both in space variables and in time we get the variational formulation of the problem (1): Find $u \in u_{0}+\mathscr{X}_{0}$ such that for every $v \in \mathscr{X}_{1}$ the following equation

$$
\begin{gathered}
-\langle\dot{u}, \dot{v}\rangle_{Q}+\langle\mathscr{A} \dot{u}, v\rangle_{Q}+\langle\mathscr{B} u, v\rangle_{Q}+\langle\mathscr{E} u, v\rangle_{Q}-\langle p(u+g), v\rangle_{Q}+\langle\dot{u}(T, \cdot), v(T, \cdot)\rangle_{\Omega} \\
=\langle f, v\rangle_{Q}+\left\langle u_{1}, v(0, \cdot)\right\rangle_{\Omega}
\end{gathered}
$$

holds. For an approximate problem $p$ is replaced by $p_{k}$ and the integration by parts in time for the acceleration term is omited, hence it is sufficient to take the test functions from $\mathscr{X}_{0}$.

In the sequel we shall assume that the operator $\mathscr{E} \equiv\{E(t) ; t \in I\}: \mathscr{X} \rightarrow \mathscr{X}^{*}$ is completely continuous, or such that $v \mapsto\langle\mathscr{E} v, v\rangle_{Q}$ is weakly lower semicontinuous on $\mathscr{X}_{0}$, or such that if a sequence $v_{k} \rightarrow v$ in $\mathscr{X}$ and $\dot{v}_{k} \rightarrow \dot{v}$ in $L_{2}(Q)$, then $\left\langle\mathscr{E} v_{k}, v_{k}\right\rangle_{Q} \rightarrow\langle\mathscr{E} v, v\rangle_{Q}$. Moreover, we assume that $\langle\mathscr{E} v, v\rangle_{Q} \geq \operatorname{const}\left(u_{0}, u_{1}\right)-c\|v\|_{\mathscr{X}}$ for $v \in \mathscr{X}$ such that $\dot{v} \in \mathscr{X}$ and the initial conditions in (11) are satisfied. Further, we assume that

$$
u_{0} \in H^{2}(Q) \text { such that } u_{0} \geq c_{0} \text { on } \bar{Q}, u_{1} \in L_{2}(\Omega) \text { and } f \in L_{2}(Q) .
$$

Here $c_{0}$ is a positive constant.

The proof of the solvability of the auxiliary problem under the assumption (3) does not differ from the proof of a penalized problem to the appropriate Signorini contact. It is solved via the Galerkin approximation using just identical arguments, because in this case the auxiliary contact term represents a completely continuous perturbation of the appropriate problem without contact. By putting $v=\left(\dot{u}_{k}-\dot{u}_{0}\right) \chi_{Q_{t}}$ in (2) with $p_{k}$, where $\chi_{M}$ is the characteristic function of a set $\mathrm{M}$ (equal 1 on $M$ and vanishing elsewhere), $t \in(0, T]$ and $Q_{t} \equiv[0, t] \times \Omega$, we get (after a certain small and obvious calculation) the a priori estimate of the respective solutions $u_{k}$ to the approximate problems with $p_{k}$

$$
\left\|\dot{u}_{k}\right\|_{L_{\infty}\left(I ; L_{2}(\Omega)\right)}^{2}+\left\|u_{k}\right\|_{L_{\infty}(I ; X)}^{2}+\left\|\dot{u}_{k}\right\|_{\mathscr{X}}^{2}+\left\|P_{k}\left(u_{k}+g\right)\right\|_{L_{\infty}\left(I ; L_{1} \Omega\right)} \leq \text { const. }
$$

where $P_{k}: s \mapsto \int_{s}^{+\infty} p_{k}(z) d z, s \in \mathbb{R}$. Let us take in mind that $L_{1}(\Omega) \subset L_{\infty}(\Omega)^{*} \hookrightarrow X^{*}$, because for the primal spaces the compact reverse embeddings hold. Since

$$
\left\|p_{k}\left(u_{k}+g\right)\right\|_{L_{1}(Q)} \leq c_{0}^{-1}\left\langle p_{k}\left(u_{k}+g\right), u_{0}-u_{k}\right\rangle_{Q}
$$

(observe that $x p_{k}(x) \leq 0, x \in \mathbb{R}$ ), the use of (2) for $v=u_{0}-u_{k}$ and the estimate (4) yields that the sequence $\left\{\left\|p_{k}\left(u_{k}+g\right)\right\|_{L_{1}(Q)}\right\}$ is bounded. Then we derive from this and (11) the dual estimate

$$
\left\|\ddot{u}_{k}\right\|_{L_{1}\left(X^{*}\right)} \leq \text { const. }
$$

with the constant independent of $k$. With the help of (5) and the classical Aubin Lemma we get a certain $u$ and $\vartheta$ such that convergences

$$
\begin{aligned}
& u_{k} \rightarrow^{*} u \text { and } \dot{u}_{k} \rightarrow^{*} \dot{u} \text { in } L_{\infty}(I ; X), L_{\infty}\left(I ; L_{2}(\Omega)\right), \text { respectively, } \dot{u}_{k} \rightarrow \dot{u} \text { in } L_{2}(Q), \\
& \left\langle\mathscr{E} u_{k}, u_{k}\right\rangle_{Q} \rightarrow\langle\mathscr{E} u, u\rangle_{Q} \text { or } \liminf _{k \rightarrow \infty}\left\langle\mathscr{E} u_{k}, u_{k}\right\rangle_{Q} \geq\langle\mathscr{E} u, u\rangle_{Q}, \text { and } p_{k}\left(u_{k}+g\right) \rightarrow \vartheta \text { in } \mathscr{X}_{1}^{*}
\end{aligned}
$$

hold for a possible subsequence. Performing the integration by parts in time for the acceleration term and putting $v=u_{k}-u_{0}$ in (2) with $p_{k}$, using the weak lower semicontinuity of the elliptic 
operators and the strong convergence of the others, we get $\langle\vartheta, u\rangle \geq \lim \sup _{k \rightarrow+\infty}\left\langle p_{k}(u)_{k}, u_{k}\right\rangle$. Since $p_{k}$ are monotone, this yields $\langle\vartheta-p(v+g), u-v\rangle \geq 0$ for every $v \in \mathscr{X}_{0}$, hence $[\vartheta, u]$ may be added to the graph of $p$ such that the extended graph remains monotone. The maximal monotonicity of $p$ proved in [5] yields that $\vartheta=p(u+g)$, hence $\mathrm{u}$ is a solution of the variational equation (21) and we are done. We have proved

Theorem 1 Under the above mentioned assumptions to the employed operators and the function $p$ there exists a solution to the problem (2).

Example 1. A biharmonic plate. Here $\mathscr{D}=\triangle, a, b$ are positive constants and $\mathscr{E}=0$.

Example 2. A von Kármán plate without rotation inertia. First we introduce for two functions $u, v$

$$
[u, v]=\partial_{11} \partial_{22} v+\partial_{22} u \partial_{11} v-2 \partial_{12} u \partial_{12} v
$$

where here and in the sequel $\partial_{i} \equiv \partial / \partial_{x_{i}}, i=1,2, \partial_{t} \equiv \partial / \partial t$ and $\partial_{i j} \equiv \partial_{i} \partial_{j}, i, j=1,2$. Then we define the bilinear operator $\Phi: H^{2}(\Omega)^{2} \rightarrow \stackrel{\circ}{H}^{2}(\Omega)$ by means of the variational equation

$$
\int_{\Omega} \triangle \Phi(u, v) \triangle \varphi d x=\int_{\Omega}[u, v] \varphi d x, u, v, \varphi \in \stackrel{\circ}{H}^{2}(\Omega) .
$$

The equation (8) has a unique solution, because $[u, v] \in L_{1}(\Omega) \hookrightarrow H^{2}(\Omega)^{*}$. The well-defined operator $\Phi$ is compact and symmetric. Let us recall Lemma 1 from [10] due to which $\Phi$ : $H^{2}(\Omega)^{2} \rightarrow W_{p}^{2}(\Omega)$, for any $p \in(2, \infty)$, and

$$
\|\Phi(u, v)\|_{W_{p}^{2}(\Omega)} \leq c\|u\|_{H^{2}(\Omega)}\|v\|_{W_{p}^{1}(\Omega)} \forall u, v \in H^{2}(\Omega)^{2},
$$

i.e. $w \mapsto \Phi(w, w)$ is completely continuous from $H^{\delta}(Q) \cap \mathscr{X}$ to $\mathscr{X}$ for any $\delta>0$.

To avoid the introduction of the Airy stress function, we introduce directly the variational formulation. For it we introduce

$$
A_{0}:\{u, y\} \mapsto b_{0}\left(\partial_{\ell \ell} u \partial_{\ell \ell} y+\nu\left(\partial_{11} u \partial_{22} y+\partial_{22} u \partial_{11} y\right)+2(1-\nu) \partial_{12} u \partial_{12} y\right), b_{0}=\text { const }>0
$$

where $\nu \in(-1 / 2,1)$ is a material constant (the Poisson ratio) and the standard summation convention for the repeating index $\ell$ is applied. Then we define $\langle\mathscr{A} \dot{u}, v\rangle_{Q}$ as $e_{1} \int_{Q} A_{0}(\dot{u}, v) d x d t$, $\langle\mathscr{B} u, v\rangle_{Q}$ as $e_{0} \int_{Q} A_{0}(u, v) d x d t, \mathscr{E}: u \mapsto b\left(\left[u, e_{1} \partial_{t} \triangle \Phi(u, u)+e_{0} \triangle \Phi(u, u)\right]\right)$, where $e_{1}, e_{0}$ are other material constants (the Young moduli) which are positive. With such defined mappings the variational formulation of the problem has exactly the form of (2). It is easy to derive that

$$
\left\langle\mathscr{E} u_{k}, u_{k}\right\rangle_{Q}=\int_{Q}\left(e_{1} / 2 \partial_{t}\left(\triangle \Phi\left(u_{k}, u_{k}\right)\right)^{2}+e_{0}\left(\triangle \Phi\left(u_{k}, u_{k}\right)\right)^{2}\right) d x d t
$$

(cf. [1]) hence it satisfies the corresponding requirements and the quadratic forms generated by such defined $\mathscr{A}, \mathscr{B},\langle\mathscr{E} \cdot, \cdot\rangle_{Q}$ are weakly lower semicontinuous and we are done. We remark that $M(u)=b\left(e_{1} m(\dot{u})+e_{0} m(u)\right)$, where $m(u)=\triangle u+(1-\nu)\left(2 n_{1} n_{2} \partial_{12} u-n_{1}^{2} \partial_{22} u-n_{2}^{2} \partial_{11} u\right)$.

Example 3, A simply supported von Kármán plate with the rotation inertia. Here the original structure (1) is enriched by the additional term $G u=g_{0} \triangle \ddot{u}$ to the right hand side of the first row of (11). If $g_{0}$ is just a positive constant, then this term contributes (after the obvious integration by parts) to the extension of the a priori estimate (4) by the term $\left\|\nabla \dot{u}_{k}\right\|_{L_{2}\left(I ; L_{2}(\Omega)\right)}^{2}$. 
The dual estimate $\left\|g \triangle \ddot{u}_{k}-\ddot{u}_{k}\right\|_{L_{2}\left(I, . X^{*}\right)} \leq$ const is here $k$-dependent. After integration by parts this gives $\sup _{v \in L_{2}(I ; X),\|v\| \leq 1}\langle\ddot{u}, g \triangle v-v\rangle_{Q} \leq$ const . The operator $g_{0} \triangle-I$, where $I$ is the identity, is an isometry between the space $X=H^{2}(\Omega) \cap \stackrel{\circ}{H}^{1}(\Omega)$ and $L_{2}(Q)$, hence the dual estimate yields $\ddot{u}_{k} \in L_{2}(Q)$. In the further treatment an additional lower semicontinuous term of this form occurs which does not change the treatment of the limit process from the approximate to the original problem. In fact from the $k$-independent $L_{1}(Q)$ estimate of the approximate contact term we get (using again the properties of the operator $\left(g_{0} \triangle-I\right)$ the $k$-independent dual estimate $\left\|\ddot{u}_{k}\right\|_{L_{1}\left(I ; L_{2}(\Omega)\right)} \leq$ const. The Aubin Lemma again yields the crucial strong convergence $\dot{u}_{k}$ to $\dot{u}$ in $L_{2}(Q)$. Hence we are in the same situation as above, $u_{k} \mapsto\left\langle p_{k}\left(u_{k}+g\right), u_{k}\right\rangle_{Q}$ is again upper semicontinuous and via the maximal monotonicity argument we are done.

\section{Von Kármán model with a singular memory}

Let us introduce the kernel $K$ of the singular memory term which is assumed to be integrable over $\mathbb{R}_{+}$and to have the form

$$
\begin{aligned}
& K: t \mapsto t^{-2 \alpha} q(t)+r(t), t \in \mathbb{R}_{+} \equiv(0,+\infty) \text { with } \alpha \in\left(0, \frac{1}{2}\right), \\
& K: t \mapsto 0, t \leq 0 .
\end{aligned}
$$

Both $q$ and $r$ belong to $C^{1}\left(\mathbb{R}_{+}\right)$; they are non-negative and non-increasing functions. Moreover, we assume that $q(t)>0$ for $t$ on an nonempty interval $\left[0, t_{0}\right]$. Let $d_{m}: v \mapsto \int_{0}^{t} K(t-s)(v(t, \cdot)-$ $v(s, \cdot)) d s$ for a function $v$ on $Q$. Let us remark that it holds

$$
\begin{aligned}
\left\langle d_{m} v, \dot{v}\right\rangle_{Q} & =\int_{Q} \int_{s}^{T} \frac{1}{2}\left(\partial_{t}\left(K(t-s)(v(t)-v(s))^{2}\right)-(v(t)-v(s))^{2} \partial_{t} K(t-s)\right) d x d t d s \\
& =\int_{Q} \frac{1}{2} K(T-s)(v(T)-v(s))^{2} d x d s-\int_{Q} \int_{0}^{t} \frac{1}{2} K^{\prime}(t-s)(v(t)-v(s))^{2} d x d t d s
\end{aligned}
$$

and the second term in this formula leads to the fractional time-derivative norm of $v$. We recall that such a norm used in the sequel is for a Banach space $X$ defined as follows:

$$
\|v\|_{H^{\alpha}(I ; X)}^{2} \equiv \int_{I}\|v\|_{X}^{2} d t+\int_{I} \int_{I} \frac{\|v(t)-v(s)\|_{X}^{2}}{|t-s|^{1+2 \alpha}} d s d t .
$$

We solve the problem

$$
\begin{gathered}
\ddot{u}-e_{1} d_{m} A u-e_{0} A u+\mathscr{E}_{0} u=f+p(u+g) \text { on } Q, \\
u=0, \quad M(u)=0 \text { on } S, u=u_{0}, \dot{u}=u_{1} \text { on } \Omega,
\end{gathered}
$$

Here $A$ is the differential operator leading to the operator defined in (10), i .e.

$$
b_{0}\left(\partial_{\ell \ell} \partial_{\ell \ell}+\nu\left(\partial_{11} \partial_{22}+\partial_{22} \partial_{11}\right)+2(1-\nu) \partial_{12} \partial_{12}\right)
$$

and

$$
\begin{aligned}
& \mathscr{E}_{0}: u \mapsto\left[u, e_{1} d_{m} \triangle \Phi(u, u)+e_{0} \triangle \Phi(u, u)\right], \\
& \left.M(u)=a d_{m} m(u)+b m(u)\right], \text { where } m(u)=\Delta u+(1-\nu)\left(2 n_{1} n_{2} \partial_{1,2} u-n_{1}^{2} \partial_{2,2} u-n_{2}^{2} \partial_{1,1} u\right) \\
& \quad \text { for a simply supported plate. } \\
& M(u)=\partial u / \partial n \text { for the clamped plate. }
\end{aligned}
$$


To be able to handle the singular memory term it is necessary to assume its smallness as follows

$$
\int_{0}^{+\infty} K(s) d s<e_{0} / 2 e_{1}
$$

which ensures that the quadratic form

$$
Z: V \mapsto \int_{Q}\left(e_{1} d_{m} V+e_{0} V\right) V d x d t, V \in L_{2}(Q)
$$

is strongly monotone.

We introduce the variational formulation of the problem. Let $X=H^{2}(\Omega) \cap \stackrel{\circ}{H}^{1}(\Omega)$ for the simply supported plate and $X=\stackrel{\circ}{H}^{2}(\Omega)$ for the clamped plate. The formulation reads: Find $u \in L_{2}(I ; X) \cap H^{1}\left(I ; H^{1}(\Omega)\right)$ such that for every $v \in L_{2}\left(I ; X_{0}\right) \cap H^{1}\left(I ; H^{1}(\Omega)\right)$ the equation

$$
\begin{gathered}
\int_{Q}\left(\left(e_{1} d_{m} A_{0}(u, y)+e_{0} A_{0}(u, y)-\dot{u} \dot{y}+\mathscr{E}_{0} u y-p(u+g) y-f y\right) d x d t\right. \\
\quad+\int_{\Omega}\left(-(\dot{u} y)(T, \cdot)+u_{1}(y(0, \cdot)) d x=0\right.
\end{gathered}
$$

holds with $A_{0}$. from (10). We shall solve this problem assuming that (3) holds.

We formulate the approximate problem again by replacing $p$ by $p_{k}$, but unlike (18) no integration by parts in time for the acceleration term is applied, hence the test function may be taken just from $\mathscr{X}_{0}$. It is solved again by the standard Galerkin procedure, for details cf. [2]. To get the $k$-independent a priori estimate for their solution, $y=\dot{u}_{k}-\dot{u}_{0}$ must be taken. After some calculation we finally obtain

$$
\begin{aligned}
& \left\|u_{k}\right\|_{H^{\alpha}\left(I ; H^{2}(\Omega)\right)}^{2}+\left\|\dot{u}_{k}\right\|_{L_{\infty}\left(I ; L_{2} 1(\Omega)\right)}^{2}+\left\|u_{k}\right\|_{L_{\infty}\left(I ; H^{2}(\Omega)\right)}^{2}+\left\|\Phi\left(u_{k}, u_{k}\right)\right\|_{H^{\alpha}\left(I ; H^{2}(\Omega)\right)}^{2} \\
& \quad+\left\|P_{k}(u+g)\right\|_{L_{\infty}\left(I ; L_{1}(\Omega)\right)} \leq c \equiv c\left(f, u_{0}, u_{1}\right)
\end{aligned}
$$

Since the singular-memory terms are remarkably weaker than the corresponding viscoelastic ones, the Aubin Lemma gives not a sufficient reasoning to prove the required strong convergence of velocities in the limit procedure for $k \rightarrow \infty$. In the sequel we shall use the following theorems:

Theorem 2 (Embedding theorem) Let $M \subset \mathbb{R}^{N}$ be a bounded domain with a Lipschitz boundary. Let $p, q \in(1, \infty), \gamma \in[0,1]$ and $\alpha \in(\gamma, 1]$ be numbers such that the inequality

$$
\frac{1}{\alpha}\left(\frac{N}{p}-\frac{N}{q}+\gamma\right) \leq 1,
$$

holds. Then the Sobolev-Slobodetskii space $W_{p}^{\alpha}(M)$ is continuously embedded into $W_{q}^{\gamma}(M)$. If inequality (20) is strict, then the embedding is compact for any real $q \geq 1$. For $q=\infty$ this is true under the convention $1 / q=0$.

Corollary 3 Let $M$ and $I$ be as above. Let $p_{i}, q_{i}$ belong to $(1,+\infty)$, $\alpha_{i}$ belong to $(0,1]$ and $\gamma_{i}$ to $\left[0, \alpha_{i}\right), i=1,2$. Assume that (20) holds with $i=1$ and $N$ replaced by 1 and that it simultaneously holds for $i=2$. Then $W_{p_{1}}^{\alpha_{1}}\left(I ; W_{p_{2}}^{\alpha_{2}}(M)\right)$ can be imbedded into $W_{q_{1}}^{\gamma_{1}}\left(I ; W_{q_{2}}^{\gamma_{2}}(M)\right)$. If both inequalities are strict, the imbedding is compact. The last assertion still holds if $q_{i}$ is infinite, provided we use the convention $1 / q_{i}=0, i=1,2$. 
Theorem 4 (Interpolation theorem) Let $M$ be as above, let $k_{1}$, $k_{2}$ belong to $[0,1]$, let $p_{1}, p_{2}$ belong to $(1,+\infty)$ and $\Theta_{\lambda}$ to $[0,1]$. Then there exists a constant c such that for all $u \in W_{p_{1}}^{k_{1}}(M) \cap$ $W_{p_{2}}^{k_{2}}(M)$ the following estimate holds

$$
\|u\|_{W_{p}^{k}(M)} \leq c\|u\|_{W_{p_{1}}^{k_{1}}(M)}^{\Theta_{1}}\|u\|_{W_{p_{2}}^{k_{2}(M)}}^{1-\Theta_{\lambda}}
$$

with $k=\Theta_{\lambda} k_{1}+\left(1-\Theta_{\lambda}\right) k_{2}$ and $\frac{1}{p}=\frac{\Theta_{\lambda}}{p_{1}}+\frac{1-\Theta_{\lambda}}{p_{2}}$. The assertion remains true if $k_{1}=k_{2}=0$ and $p_{1}, p_{2}$ belong to $[1,+\infty]$.

Corollary 5 (Generalization) Let $M, k_{1}, k_{2}, p_{1}, p_{2}$ be as above. Let $I$ be a bounded interval in $\mathbb{R}$, let $\kappa_{1}, \kappa_{2}$ belong to $[0,1]$, let $q_{1}, q_{2}$ belong to $(1,+\infty)$ and $\Theta_{\lambda}$ to $[0,1]$. Then there exists a constant $c$ such that for all $u \in W_{q_{1}}^{\kappa_{1}}\left(I ; W_{p_{1}}^{k_{1}}(M)\right) \cap W_{q_{2}}^{\kappa_{2}}\left(I ; W_{p_{2}}^{k_{2}}(M)\right)$ it holds

$$
\|u\|_{W_{q}^{k}\left(I ; W_{p}^{k}(M)\right)} \leq c\|u\|_{W_{q_{1}}^{\kappa_{1}}\left(I ; W_{p_{1}}^{k_{1}}(M)\right)}^{\Theta_{1}}\|u\|_{W_{q_{2}}^{\kappa_{2}}\left(I ; W_{p_{2}}^{k_{2}}(M)\right)}^{1-\Theta_{\lambda}},
$$

where $k=\Theta_{\lambda} k_{1}+\left(1-\Theta_{\lambda}\right) k_{2}, \kappa=\Theta_{\lambda} \kappa_{1}+\left(1-\Theta_{\lambda}\right) \kappa_{2}, \frac{1}{q}=\frac{\Theta_{\lambda}}{q_{1}}+\frac{1-\Theta_{\lambda}}{q_{2}}$ and $\frac{1}{p}=\frac{\Theta_{\lambda}}{p_{1}}+\frac{1-\Theta_{\lambda}}{p_{2}}$. If $\kappa_{1}=\kappa_{2}=0$ and $q_{1}, q_{2}$ belong to $[1,+\infty]$, the assertion still holds.

The proofs of the above mentioned facts follows from Chapter 2 of the monograph [6].

The assumed smallness of the memory term yields again the uniform estimate of $\left\{\| p_{k}(u+\right.$ $\left.g) \|_{L_{1}(Q)}\right\}$ which leads to the dual estimate $\left\|\ddot{u}_{k}\right\|_{L_{1}\left(I ; X^{*}\right)} \leq$ const. Hence $\left\{\dot{u}_{k}\right\}$ is bounded in $W_{1+\varepsilon_{2}}^{1-\varepsilon_{1}}\left(I ; H^{-2-\varepsilon_{3}}(\Omega)\right)$ for any $\varepsilon_{2}>0, \varepsilon_{3}>0$ and for $\varepsilon_{1} \equiv \varepsilon_{1}\left(\varepsilon_{2}\right) \searrow 0$ if $\varepsilon_{2} \searrow 0$. Interpolating this space with the space $L_{q}\left(I ; L_{2}(\Omega)\right)$ for $q=1+1 / \varepsilon_{2}$ we get that

$$
\left\|\dot{u}_{k}\right\|_{H^{1 / 2}\left(I ; H^{-1-\theta}(\Omega)\right)} \leq C \text {, i. e. }\left\|u_{k}\right\|_{H^{3 / 2}\left(I ; H^{-1-\theta}(\Omega)\right)} \leq C \text { with } 0<\theta \text { arbitrarily small. }
$$

Interpolating the result in (21) with the fact that $\left\{u_{k}\right\}$ is bounded in $H^{\alpha}\left(I ; H^{2}(\Omega)\right)$, we get that $\left\{\dot{u}_{k}\right\}$ is bounded in $H^{\theta_{1}}\left(I ; L_{2}(\Omega)\right)$ for $\theta_{1}, \in(0, \alpha / 3)$. Interpolation of this space with the time-fractional derivative space from (19) gives the space $L_{2}\left(I ; H^{\delta_{2}}\right)$ with $\delta_{2} \in(0,2 \alpha /(3-2 \alpha))$, hence $\left\{\dot{u}_{k}\right\}$ is bounded in the anizotropic space $H^{\theta_{1}, \theta_{2}}(Q)$. This space is compactly imbedded into $L_{2}(Q)$ which ensures that $\left\langle\dot{u}_{k}, \dot{u}_{k}\right\rangle_{Q}$ tends strongly to the limit $\langle\dot{u}, \dot{u}\rangle_{Q}$ even for the weak convergence of $u_{k}$ in the employed spaces. Similarly to (11) we can derive that

$$
\left\langle\mathscr{E}_{0} u_{k}, u_{k}\right\rangle_{Q}=\int_{Q}\left(e_{1} \triangle d_{m} \Phi\left(u_{k}, u_{k}\right) \triangle \Phi\left(u_{k}, u_{k}\right)+e_{0}\left(\triangle \Phi\left(u_{k}, u_{k}\right)\right)^{2}\right) d x d t
$$

The compactness of $\Phi$ based on (9) and the fractional time-derivative norm in (19) yield the needed strong convergence of this term. Hence we are able for the limit procedure $k \rightarrow+\infty$ to prove again the upper semicontinuity of $\left\langle p_{k}\left(u_{k}\right), u_{k}\right\rangle_{Q}$ and with the maximal monotonicity argument to prove $p_{k}\left(u_{k}+g\right) \rightarrow p(u+g)$. Thus $u$ is a solution of (18) and with the additional assumption (16) the existence theorem is proved also for this problem.

\section{The problem for more complex viscoelastic plate mod- els}

In this section we shall treat the Reissner-Mindlin plate model as well as full von Kármán system. The plates are again in contact with the limited interpenetration with a foundation. 


\subsection{Contact of Reissner-Mindlin plates}

In this 2 nd order model besides the vertical deflection $u$ there is the $2 \mathrm{D}$-vector $\boldsymbol{\varphi}$ of angles of rotations of the cross sections of the plate. We denote by $\mathbb{S}$ the set of symmetric $2 \times 2$ tensors with the product $\boldsymbol{\kappa} \odot \boldsymbol{\lambda}=\kappa_{i j} \lambda_{i j}$, where the Einstein summation convention (summing over repeated indices) is employed. Moreover for $\omega \equiv\left\{\omega_{i j}, i, j=1,2\right\} \in \mathbb{S}$ we denote Div $\omega \equiv\left(\partial_{i} \omega_{1 i}, \partial_{i} \omega_{2 i}\right)$ and $\operatorname{tr} \omega=\omega_{11}+\omega_{22}$.

With the notation

$$
\begin{aligned}
J(u, \boldsymbol{\varphi}) & =e_{1}(\nabla \dot{u}+\dot{\boldsymbol{\varphi}})+e_{0}(\nabla u+\boldsymbol{\varphi}), \\
\mathscr{C}_{i}(\omega) & =\frac{\tilde{c}}{\left(1-\nu_{i}^{2}\right)}\left(\nu_{i}(\operatorname{tr} \omega) I_{\mathbb{S}}+\left(1-\nu_{i}\right) \omega\right), \omega \in \mathbb{S}, i=0,1,
\end{aligned}
$$

where $I_{\mathbb{S}}$ is the unit matrix in $\mathbb{S}, \tilde{c}, e_{0}, e_{1}$ are given positive constants, and the Poisson ratio $\nu_{i} \in(-1,1 / 2), i=0,1$ the classical formulation of the viscoelastic ("short memory") problem is as follows: We look for $(u, \varphi)$ such that the system

$$
\left.\begin{array}{rl}
\ddot{u}-\operatorname{div} J(u, \boldsymbol{\varphi}) & =f+p(u+g), \\
\ddot{\varphi}-\operatorname{Div}\left(\mathscr{C}_{1}\left(\varepsilon_{0}(\dot{\boldsymbol{\varphi}})\right)+\mathscr{C}_{0}\left(\varepsilon_{0}(\boldsymbol{\varphi})\right)+J(u, \boldsymbol{\varphi})\right. & =\boldsymbol{M}
\end{array}\right\} \text { on } Q,
$$

the boundary value conditions

$$
\left.\begin{array}{rl}
u=u_{0}, \boldsymbol{\varphi}=\mathbf{0} & \text { for a clamped plate } \\
u=u_{0}, \quad\left(\mathscr{C}_{1}\left(\boldsymbol{\varepsilon}_{0}(\dot{\boldsymbol{\varphi}})\right)+\mathscr{C}_{0} \boldsymbol{\varepsilon}_{0}(\boldsymbol{\varphi})\right) \cdot \boldsymbol{n}=\mathbf{0} & \text { for a simply supported one }
\end{array}\right\} \text { on } S,
$$

and the initial conditions

$$
\left.\begin{array}{l}
u(0, \cdot)=u_{0}, \quad \dot{u}(0, \cdot)=u_{1}, \\
\boldsymbol{\varphi}(0, \cdot)=\varphi^{(0)}, \dot{\varphi}(0, \cdot)=\varphi^{(1)}
\end{array}\right\} \text { on } \Omega
$$

are satisfied. Here $\boldsymbol{\varepsilon}_{0}$ is the standard 2D linearized strain tensor and $\boldsymbol{n}$ is the unit outer normal vector. We assume that the function $p$ satisfies all the assumptions listed at the beginning of Section 2, we assume that (3) still holds, in particular the positive function $u_{0}$ is again bounded away from 0 . Moreover, we assume that $\boldsymbol{\varphi}^{(1)} \in \boldsymbol{L}_{2}(\Omega), \boldsymbol{\varphi}^{(0)} \in \boldsymbol{H}^{1}(\Omega)$ and $\boldsymbol{M} \in \boldsymbol{L}_{2}(Q)$.

The variational formulation of the problem based on appropriate integrations by parts has the following form: Look for $\{u, \varphi\} \in\left(u_{0}+L_{2}\left(I ; \stackrel{\circ}{H}^{1}(\Omega)\right) \times \boldsymbol{X}(Q)\right.$ such that $\dot{u} \in L_{2}\left(I ; H^{1}(\Omega)\right), \dot{\varphi} \in$ $L_{2}(I ; \boldsymbol{X}(\Omega)), \ddot{\boldsymbol{\varphi}} \in \boldsymbol{L}^{2}(Q)$, the first condition in the first row and the second row of the initial conditions (25) are satisfied and the system

$$
\begin{aligned}
& \int_{Q}(J(u, \boldsymbol{\varphi}) \cdot \nabla y-\dot{u} \dot{y}-p(u+g) y) d x d t=\int_{\Omega}\left(u_{1} y(0, \cdot)-\dot{u}(T, \cdot) y(T, \cdot)\right) d x+\int_{Q} f y d x d t \\
& \int_{Q}\left(\ddot{\boldsymbol{\varphi}} \cdot \boldsymbol{\psi}+\left(\mathscr{C}_{1}\left(\boldsymbol{\varepsilon}_{0}(\dot{\boldsymbol{\varphi}})\right)+\mathscr{C}_{0}\left(\boldsymbol{\varepsilon}_{0}(\boldsymbol{\varphi})\right)\right) \odot \boldsymbol{\varepsilon}_{0}(\boldsymbol{\psi})+J(u, \boldsymbol{\varphi}) \cdot \boldsymbol{\psi}\right) d x d t=\int_{Q} \boldsymbol{M} \cdot \boldsymbol{\psi} d x d t
\end{aligned}
$$

holds for any $\{y, \boldsymbol{\psi}\} \in \stackrel{\circ}{H}^{1}(Q) \times L_{2}(I, \boldsymbol{X}(\Omega))$. Here $\boldsymbol{X}$ stands for $\stackrel{\boldsymbol{H}}{ }^{1}, \boldsymbol{H}^{1}$ for clamped and simply supported plates, respectively.

As in previous cases we introduce the approximate problems by replacing the original function $p$ by the approximate function $p_{k}$ and the integration by parts in time for the acceleration term in the first row of (26) is omitted. Hence it has the form

$$
\int_{Q}\left(J\left(u_{k}, \boldsymbol{\varphi}_{k}\right) \cdot \nabla y+\ddot{u}_{k} y\right) d x d t=\int_{Q}\left(f+p_{k}\left(u_{k}+g\right)\right) y d x d t .
$$


We put $\{y, \boldsymbol{\psi}\}=\left\{\dot{u}_{k}-\dot{u}_{0}, \dot{\boldsymbol{\varphi}}_{k}\right\}$ as the test function of the approximate system and integrate on the interval $[0, s], s \leq T$. Adding both lines of (26) and using the standard integration by parts we get

$$
\begin{gathered}
\int_{Q_{s}}\left(\frac{1}{2} \partial_{t}\left(\dot{u}_{k}^{2}+d_{0}\left|\nabla u_{k}+\boldsymbol{\varphi}_{k}\right|^{2}+\left|\dot{\boldsymbol{\varphi}}_{k}\right|^{2}+\mathscr{C}_{0}\left(\boldsymbol{\varepsilon}_{0}\left(\boldsymbol{\varphi}_{k}\right)\right) \odot \boldsymbol{\varepsilon}_{0}\left(\boldsymbol{\varphi}_{k}\right)+P_{k}\left(u_{k}+g\right)\right)\right. \\
\left.+d_{1}\left|\nabla \dot{u}_{k}+\dot{\boldsymbol{\varphi}}_{k}\right|^{2}+\mathscr{C}_{1}\left(\boldsymbol{\varepsilon}_{0}\left(\dot{\boldsymbol{\varphi}}_{k}\right)\right) \odot \boldsymbol{\varepsilon}_{0}\left(\dot{\boldsymbol{\varphi}}_{k}\right)\right) d x d t=\int_{Q_{s}}\left(f \dot{u}_{k}+\boldsymbol{M} \dot{\boldsymbol{\varphi}}_{k}\right) d x d t \\
+\int_{Q_{s}} R\left(\dot{u}_{0}\right) d x d t
\end{gathered}
$$

where in $R\left(u_{0}\right)$ we sum up all the terms containing $\dot{u}_{0}$, or its derivatives. From the positive definiteness of the tensors $\mathscr{C}_{i}$ and the last identity we derive after some calculation the a priori estimate

$$
\begin{aligned}
& \left\|\dot{u}_{k}\right\|_{L_{\infty}\left(I ; L_{2}(\Omega)\right)}^{2}+\left\|\dot{\boldsymbol{\varphi}}_{k}\right\|_{L_{\infty}\left(I ; \boldsymbol{L}_{2}(\Omega)\right)}+\left\|\dot{u}_{k}\right\|_{L_{2}\left(I ; H^{1}(\Omega)\right)}^{2}+\left\|\dot{\boldsymbol{\varphi}}_{k}\right\|_{L_{2}\left(I ; \boldsymbol{H}^{1}(\Omega)\right)}^{2}+\left\|u_{k}\right\|_{C\left(\bar{I} ; H^{1}(\Omega)\right)}^{2} \\
& +\left\|\boldsymbol{\varphi}_{k}\right\|_{C\left(\bar{I} ; \boldsymbol{H}^{1}(\Omega)\right)}^{2}+\left\|P_{k}\left(u_{k}+g\right)\right\|_{L_{\infty}\left(I ; L_{1}(\Omega)\right)} \leq c \equiv c\left(f, \boldsymbol{M}, u_{0}, u_{1}, \boldsymbol{\varphi}^{(0)}, \boldsymbol{\varphi}^{(1)}\right) .
\end{aligned}
$$

Observe that this estimate is $k$-independent.

We continue with the estimates of the acceleration terms. After using $\left\{\ddot{u}_{k}-\ddot{u}_{0}, \ddot{\varphi}_{k}\right\}$ as the test function we obtain

$$
\begin{aligned}
& \left\|\ddot{\boldsymbol{\varphi}}_{k}\right\|_{L_{2}(Q)}^{2} \leq c, \\
& \left\|\ddot{u}_{k}\right\|_{L_{2}(Q)}^{2} \leq c_{k}, \quad k \in \mathbb{N} .
\end{aligned}
$$

From (29) it is easy to see that (30) is again $k$-independent. However, (31) depends on $k$ and for the limit process $k \rightarrow \infty$ it must be replaced by the dual estimate of $\ddot{u}_{k}$ based on the estimate of the contact term.

These approximate problems are solved by means of the Galerkin approximation. Since they do not structurally differ from the penalized problems for the Signorini contact (in both described cases the approximate contact term represents a compact perturbation of the noncontact problems) and we are focused here on the difference between the rational contact with limited interpenetration and the Signorini contact, we omit details of this well-known process here and postpone the readers to [4] for them.

To derive the crucial dual estimate of $\ddot{u}_{k}$ we can use the general abstract approach of Section 2. However, the space $H^{1}(\Omega)$ is not imbedded into $L_{\infty}(\Omega)$, hence we must use $X=$ $H^{1}(\Omega) \cap L_{\infty}(\Omega)$ here. The resulting estimate (5) yields the required strong convergence of $\dot{u}_{k}$ in $L_{2}(Q)$ in the process $k \rightarrow \infty$ via the Aubin Lemma. We put $\{y, \boldsymbol{\psi}\}=\left\{u_{k}-u_{0}, \boldsymbol{\varphi}_{k}\right\}$ in (26) and add both equations. We get

$$
\begin{gathered}
\int_{Q_{T}}\left(-\dot{u}_{k}^{2}+e_{0}\left|\nabla u_{k}+\boldsymbol{\varphi}_{k}\right|^{2}+\left|\dot{\boldsymbol{\varphi}}_{k}\right|^{2}+\mathscr{C}_{0}\left(\boldsymbol{\varepsilon}_{0}\left(\boldsymbol{\varphi}_{k}\right)\right) \odot \boldsymbol{\varepsilon}_{0}\left(\boldsymbol{\varphi}_{k}\right)+p_{k}\left(u_{k}+g\right)\left(u_{k}-u_{0}\right)\right) \\
+\partial_{t}\left(e_{1}\left|\nabla u_{k}+\boldsymbol{\varphi}_{k}\right|^{2}+\mathscr{C}_{1}\left(\boldsymbol{\varepsilon}_{0}\left(\boldsymbol{\varphi}_{k}\right)\right) \odot \boldsymbol{\varepsilon}_{0}\left(\boldsymbol{\varphi}_{k}\right)\right) d x d t=\int_{Q_{T}}\left(f \dot{u}_{k}+\boldsymbol{M} \dot{\boldsymbol{\varphi}}_{k}\right) d x d t \\
+\int_{Q_{T}} R_{1}\left(u_{0}, u_{1}\right) d x d t
\end{gathered}
$$

where $R_{1}$ contains all remaining terms. Obviously they contain $u_{0}$ or $u_{1}$ or their derivatives. This identity shows again that it belongs to the abstract structure described in Section 2. Besides weakly lower semicontinuous elliptic terms and weakly continuous terms as $\dot{u}_{k}^{2}$ and $R_{1}$ the 
only remaining term, the contact one, must be upper semicontinuous and we can use again the maximal monotonicity argument for it to prove $p_{k}\left(u_{k}+g\right) \rightarrow p(u+g)$. Hence the limit $u$ of the sequence $\left\{u_{k}\right\}$ satisfies (26) and we are done.

In the classical formulation of the Reissner-Mindlin plate with a singular memory we replace all the "short memory" terms in $J$ and $\mathscr{C}_{1}$ (i.e. the terms containing the time derivatives) by the corresponding singular memory terms (the $d_{m}$ versions of the elastic terms), where we use again the kernel $K$ defined in (12). Hence $J(u, \boldsymbol{\varphi}) \equiv e_{0}(\nabla u+\boldsymbol{\varphi})+e_{1} d_{m}(\nabla u+\boldsymbol{\varphi})$. With this modification the structure of (23), (24), and (25) remains preserved. We assume again the sufficient smallness of the memory. To get it exactly in the form (16) we assume $\nu_{1}=\nu_{0}$.

We present explicitely its variational formulation which reads: Look for $\{u, \varphi\} \in\left(u_{0}+\right.$ $\left.\stackrel{\circ}{H}^{1}(Q)\right) \times L_{2}(I ; \boldsymbol{X}(\Omega))$ such that $\ddot{\boldsymbol{\varphi}} \in \boldsymbol{L}^{2}(Q)$, the first condition in the first row and the second row of (25) are satisfied and the system

$$
\begin{aligned}
& \int_{Q}(J(u, \boldsymbol{\varphi}) \cdot \nabla y-\dot{u} \dot{y}) d x d t=\int_{\Omega}\left(u_{1} y(0, \cdot)-\dot{u}(T, \cdot) y(T, \cdot)\right) d x+\int_{Q}(f+p(u+g)) y d x d t \\
& \int_{Q}\left(\ddot{\boldsymbol{\varphi}} \cdot \boldsymbol{\psi}+\left(\mathscr{C}_{1}\left(d_{m} \boldsymbol{\varphi}\right)+\mathscr{C}_{0}(\boldsymbol{\varphi})\right) \odot \boldsymbol{\varepsilon}_{0}(\boldsymbol{\psi})+J(u, \boldsymbol{\varphi}) \cdot \boldsymbol{\psi}\right) d x d t=\int_{Q} \boldsymbol{M} \cdot \boldsymbol{\psi} d x d t
\end{aligned}
$$

holds for any $\{y, \boldsymbol{\psi}\} \in L_{2}\left(I ; H^{1}(\Omega)\right) \times L_{2}(I, \boldsymbol{X}(\Omega))$. Here again $\boldsymbol{X}$ stands for $\stackrel{H}{ }^{1}, \boldsymbol{H}^{1}$ for clamped and simply supported plates, respectively.

We formulate again the approximate problems by replacing the function $p$ by $p_{k}$ and by omitting the integration by parts at the acceleration term. We solve this problem via the Galerkin method as usually. We again omit here the details postponing the readers to the paper [4]. Since it is not clear at the beginning whether the velocity $\dot{u}_{k}$ possesses the requred qualites of the test function, the apriori estimates there have been derived for the finite-dimensional space approximations and then the limit process to the original infinite-dimensional space has been performed. However, the result is the same as if we put formally $\left\{\dot{u}_{k}, \dot{\varphi}_{k}\right\}$ as the test function.

Summing up both equations and limiting the integration to the cylinder $Q_{s}$ for $s \leq T$ we obtain using the properties of the kernel function $K$ the identity

$$
\begin{aligned}
\int_{Q_{s}}( & \frac{1}{2} \partial_{t}\left(\dot{u}_{k}^{2}+d_{0}\left|\nabla u_{k}+\boldsymbol{\varphi}_{k}\right|^{2}+\left|\dot{\boldsymbol{\varphi}}_{k}\right|^{2}+\mathscr{C}\left(\boldsymbol{\varepsilon}_{0}\left(\boldsymbol{\varphi}_{k}\right)\right) \odot \boldsymbol{\varepsilon}_{0}\left(\boldsymbol{\varphi}_{k}\right)+P_{k}\left(u_{k}+g\right)\right) \\
& +\frac{d}{2} K(s-t)\left|\nabla\left(u_{k}(s)-u_{k}(t)\right)+\boldsymbol{\varphi}_{k}(s)-\boldsymbol{\varphi}_{k}(t)\right|^{2} \\
& +\frac{b}{2} K(s-t) \mathscr{C}\left(\varepsilon_{0}\left(\boldsymbol{\varphi}_{k}(s)-\boldsymbol{\varphi}_{k}(t)\right) \odot \boldsymbol{\varepsilon}_{0}\left(\boldsymbol{\varphi}_{k}(s)-\boldsymbol{\varphi}_{k}(t)\right)\right) d x d t \\
& -\frac{d}{2} \int_{Q_{s}} \int_{0}^{t} K_{t}^{\prime}(t-\tau)\left|\nabla\left(u_{k}(t)-u_{k}(\tau)\right)+\boldsymbol{\varphi}_{k}(t)-\boldsymbol{\varphi}_{k}(\tau)\right|^{2} d \tau d x d t \\
& -\frac{b}{2} \int_{Q_{s}} \int_{0}^{t} K_{t}^{\prime}(t-\tau) \mathscr{C}\left(\boldsymbol{\varepsilon}_{0}\left(\boldsymbol{\varphi}_{k}(t)-\boldsymbol{\varphi}_{k}(\tau)\right) \odot \boldsymbol{\varepsilon}_{0}\left(\boldsymbol{\varphi}_{k}(t)-\boldsymbol{\varphi}_{k}(\tau)\right) d \tau d x d t\right. \\
& =\int_{Q_{s}}\left(f \dot{u}_{k}+\boldsymbol{M} \dot{\boldsymbol{\varphi}}_{k}\right) d x d t .
\end{aligned}
$$

By virtue of (12), (16) the identity (34) leads to the a priori estimates independent of of $k \in \mathbb{N}$ :

$$
\begin{aligned}
& \left\|\dot{u}_{k}\right\|_{L_{\infty}\left(I ; L_{2}(\Omega)\right)}^{2}+\left\|\dot{\boldsymbol{\varphi}}_{k}\right\|_{L_{\infty}\left(I ; \boldsymbol{L}_{2}(\Omega)\right)}^{2}+\left\|u_{k}\right\|_{H^{\alpha}\left(I ; H^{1}(\Omega)\right)}^{2}+\left\|\boldsymbol{\varphi}_{k}\right\|_{H^{\alpha}\left(I ; \boldsymbol{H}^{1}(\Omega)\right)}^{2}+\left\|u_{k}\right\|_{L_{\infty}\left(I ; H^{1}(\Omega)\right)}^{2} \\
& +\left\|\boldsymbol{\varphi}_{k}\right\|_{L_{\infty}\left(I ; \boldsymbol{H}^{1}(\Omega)\right)}^{2}+\left\|P_{k}\left(u_{k}+g\right)\right\|_{L_{\infty}\left(I ; L_{1}(\Omega)\right)} \leq c \equiv c\left(f, \boldsymbol{M}, u^{(0)}, u^{(1)}, \boldsymbol{\varphi}^{(0)}, \boldsymbol{\varphi}^{(1)}\right) .
\end{aligned}
$$


The estimate of the accelerations is a straightforward consequence of the a priori estimate (35) and the approximate system to (33) and has the form:

$$
\begin{gathered}
\left\|\ddot{\boldsymbol{\varphi}}_{k}\right\|_{\boldsymbol{L}_{2}\left(I ;\left(H^{1}(\Omega)\right)^{*}\right)}^{2} \leq c, \\
\left\|\ddot{u}_{k}\right\|_{L_{2}\left(I ;\left(H^{1}(\Omega)\right)^{*}\right)}^{2} \leq c_{k}
\end{gathered}
$$

(the first of them is again $k$-independent).

For the limit process $k \rightarrow \infty$ we can get the dual estimate $\left\|\ddot{u}_{k}\right\|_{L_{1}\left(I ; H^{-1-\tilde{\varepsilon}}(\Omega)\right)} \leq$ const. via the $L_{1}$ estimate of the approximate contact term (cf. (5) ) if we employ the dual embedding $H^{1+\tilde{\varepsilon}}(\Omega) \hookrightarrow L_{\infty}(\Omega)$ for any $\tilde{\varepsilon}>0$. Then the sequence $\left\{\dot{u}_{k}\right\}$ is bounded in $W_{1+\varepsilon_{2}}^{1-\varepsilon_{1}}\left(I ; H^{-1-\tilde{\varepsilon}}(\Omega)\right)$ for any $\varepsilon_{2}>0$ and $\varepsilon_{1} \equiv \varepsilon_{1}\left(\varepsilon_{2}\right) \searrow 0$ if $\varepsilon_{2} \searrow 0$. Simultaneously it is bounded in $L_{q}\left(I ; L_{2}(\Omega)\right)$ for any $q \geq 2$. After interpolating the spaces $W_{1+\varepsilon_{2}}^{1-\varepsilon_{1}}\left(I ; H^{-1-\varepsilon_{0}}(\Omega)\right)$ and $L_{q}\left(I ; L_{2}(\Omega)\right)$ for $q \geq 1+1 / \varepsilon_{2}$ we have

$$
\left\|\dot{u}_{k}\right\|_{H^{1 / 2}\left(I ; H^{\left.-1 / 2-\varepsilon_{3}(\Omega)\right)}\right.} \leq \text { const., i. e. }\left\|u_{k}\right\|_{H^{3 / 2}\left(I ; H^{\left.-1 / 2-\varepsilon_{3}(\Omega)\right)}\right.} \leq \text { const., } k \in \mathbb{N},
$$

where $\varepsilon_{3}>0$ is arbitrarily small.. Interpolating this result with the fact that $\left\{u_{k}\right\}$ is bounded in $H^{\alpha}\left(I ; H^{1}(\Omega)\right)$ for the given $\alpha \in\left(0, \frac{1}{2}\right)$, we obtain that $\left\{u_{k}\right\}$ is bounded in the space $H^{1+\delta_{1}}\left(I ; L_{2}(\Omega)\right)$ for any $\delta_{1} \in(0, \alpha / 3)$. Interpolating this result with the same space we get the boundedness of $\left\{u_{k}\right\}$ in $H^{1}\left(I ; H^{\delta_{2}}(\Omega)\right)$ for any $\delta_{2} \in(0, \alpha /(3-2 \alpha))$. The intersection of both resulting spaces is obviously compactly imbedded into $H^{1}\left(I ; L_{2}(\Omega)\right)$, hence the strong convergence of the velocities is proved. As earlier, the resulting upper semicontinuity of the contact term and the maximal monotonicity argument for $p$ leads to the fact that $p_{k}\left(u_{k}+g\right) \rightarrow p(u+g)$ and the existence of a solution to the system (33) is thus ensured.

\subsection{Contact of viscoelastic plates described by full von Kármán sys- tem}

This model of plates describes the vertical deflection $u$ as well as the horizontal ones denoted by $\boldsymbol{u} \equiv\left\{u_{1}, u_{2}\right\}$. We assume that the potential contact is both with the foundation of the plate and on the boundary $\Gamma$. We preserve the notation of $\mathscr{C}_{i}$ from (22), but the physical meaning of some terms may differ here from the previous parts. Denoting

$$
\mathfrak{C}_{0}=e_{0} \mathscr{C}_{0}(\varepsilon(\boldsymbol{u})+\Psi(\nabla u)), \mathfrak{C}_{1}=e_{1} \mathscr{C}_{1}\left(\varepsilon(\dot{\boldsymbol{u}})+\partial_{t} \Psi(\nabla u)\right), \Psi(\boldsymbol{a})=\frac{1}{2} \boldsymbol{a} \otimes \boldsymbol{a}, \boldsymbol{a} \in \mathbb{R}^{2},
$$

we state the classical formulation of the problem:

We look for $\{\boldsymbol{u}, u\}$ such that the system

$$
\left.\begin{array}{l}
\ddot{\boldsymbol{u}}-\operatorname{Div}\left(\mathfrak{C}_{1}+\mathfrak{C}_{0}\right)=\boldsymbol{F}, \\
\ddot{u}-a \triangle \ddot{u}+b\left(e_{1} \triangle^{2} \dot{u}+e_{0} \triangle^{2} u\right)-\operatorname{div}\left(\left(\mathfrak{C}_{1}+\mathfrak{C}_{0}\right) \nabla u\right)=f+p(u+g)
\end{array}\right\} \text { on } Q
$$

holds, the boundary value conditions

$$
\left.\begin{array}{r}
\left(\mathfrak{C}_{1}+\mathfrak{C}_{0}\right) \boldsymbol{n} \cdot \boldsymbol{n}=\tilde{q}\left(\widetilde{u_{n}}\right),\left(\mathfrak{C}_{1}+\mathfrak{C}_{0}\right) \boldsymbol{n} \cdot \boldsymbol{\tau}=0, u=u^{(0)} \\
e_{1}\left(\triangle \dot{u}+\left(1-\nu_{1}\right) B \dot{u}\right)+e_{0}\left(\triangle u+\left(1-\nu_{0}\right) B u\right)=0
\end{array}\right\} \text { on } S
$$

with

$$
\widetilde{u_{n}} \equiv \boldsymbol{u} \cdot \boldsymbol{n}, B w=2 n_{1} n_{2} \partial_{12} w-n_{1}^{2} \partial_{22} w-n_{2}^{2} \partial_{11} w
$$


are satisfied, and the initial conditions

$$
\boldsymbol{u}(0, \cdot)=\boldsymbol{u}^{(0)}, \dot{\boldsymbol{u}}(0, \cdot)=\boldsymbol{u}^{(1)}, u(0, \cdot)=u^{(0)}, \dot{u}(0, \cdot)=u^{(1)} \text { on } \Omega
$$

are valid. Both functions $p$ and $-\tilde{q}$ are assumed to satisfy all the conditions to $p$ in Sec. 2 . We remark that we assume our plate to be simply supported, because it does not seem physically reasonable to consider the clamped plate with the possible limited interpenetration on the boundary. The constants $a, b, e_{0}, e_{1}$ are positive, the nonnegative function $g$ is again the gap function. Of course, we can introduce another gap function to the boundary contact, but it seems to have a little use in practical applications. Let us remark that such defined problem describes the behaviour of a cover of a fully recessed stack.

For $z, y \in L_{2}\left(I ; H^{2}(\Omega)\right)$ we define the following bilinear forms:

$$
A_{i}:(z, y) \mapsto b e_{i}\left(\partial_{k k} z \partial_{k k} y+\nu_{i}\left(\partial_{11} z \partial_{22} y+\partial_{22} z \partial_{11} y\right)+2\left(1-\nu_{i}\right) \partial_{12} z \partial_{12} y\right), i=0,1
$$

Then our problem has the following variational formulation:

Look for $\{\boldsymbol{u}, u\} \in \boldsymbol{H}^{1}(Q) \times\left(L_{2}\left(I ; H^{2}(\Omega)\right) \cap L_{2}\left(I ; \stackrel{\circ}{H}^{1}(\Omega)\right)\right)$ such that $\dot{\boldsymbol{u}} \in L_{2}\left(I ; \boldsymbol{H}^{1}(\Omega)\right), \dot{u} \in$ $L_{2}\left(I ; H^{2}(\Omega)\right)$, and the system

$$
\begin{aligned}
& \int_{Q}\left(\left(\mathfrak{C}_{1}+\mathfrak{C}_{0}\right) \varepsilon(\boldsymbol{y})-\dot{\boldsymbol{u}} \cdot \dot{\boldsymbol{y}}\right) d x d t+\int_{\Omega}\left((\dot{\boldsymbol{u}} \cdot \boldsymbol{y})(T, .)-\boldsymbol{u}^{1} \cdot \boldsymbol{y}(0, .)\right) d x \\
& =\int_{Q} \boldsymbol{F} \cdot \boldsymbol{y} d x d t-\int_{S} q\left(\widetilde{u_{n}}\right) y_{n} d x_{s} d t \\
& \left.\int_{Q}\left(A_{1}(\dot{u}, z)+A_{0}(u, z)+\left[\left(\mathfrak{C}_{1}+\mathfrak{C}_{0}\right) \nabla u\right] \cdot \nabla z\right)-\dot{u} \dot{z}-a \nabla \dot{u} \cdot \nabla \dot{z}\right) d x d t+ \\
& \int_{\Omega}\left((\dot{u} z+a \nabla \dot{u} \cdot \nabla z)(T, \cdot)-u^{(1)} z(0, \cdot)-a \nabla u^{(1)} \cdot \nabla z(0, \cdot)\right) d x=\int_{Q}(f+p(u+g)) z d x d t
\end{aligned}
$$

is satisfied for every $\{\boldsymbol{y}, z\} \in \boldsymbol{Y}$ with

$$
\begin{aligned}
\boldsymbol{Y} \equiv \boldsymbol{Y}_{0} \cap \boldsymbol{Y}_{d} \text { with } \boldsymbol{Y}_{0} & \equiv\left\{L_{2}\left(I ; \boldsymbol{H}^{1}(\Omega)\right) \times\left(L_{2}\left(I ; \stackrel{\circ}{ }^{1}(\Omega)\right) \cap L_{2}\left(I ; H^{2}(\Omega)\right)\right)\right\} . \\
\boldsymbol{Y}_{d} & \equiv\left\{\boldsymbol{z} \in \boldsymbol{Y}_{0} ; \dot{\boldsymbol{z}} \in \boldsymbol{L}_{2}(Q)\right\} .
\end{aligned}
$$

The approximate problems are defined as usually by replacing $p, q$ by $p_{k}, q_{k}$, respectively, and keeping acceleration terms in such modified system (43) in their original form. Since this problem is remarkably more complex that all previous ones and leads to more complex formulae, we shall denote the solution of this problem also by $\{\boldsymbol{u}, u\}$. Similarly to the previous problems this approximate problem is again solved with the help of the Galerkin approximation. Since there is no substantial difference between it and the penalized problem treated in [3], we postpone the readers to that paper for details. To derive a priori estimates for the solutions of the approximate problem we put $\chi_{Q_{s}}\left\{\dot{\boldsymbol{u}}, \dot{u}-\dot{u}^{(0)}\right\}$ for $s \in(0, T]$ as a test function of the appropriate variant of (43). We obtain after the integration and the summation

$$
\begin{aligned}
& \int_{Q_{s}}\left(\frac{1}{2} \partial_{t}\left(\dot{u}^{2}+a|\nabla \dot{u}|^{2}+|\dot{\boldsymbol{u}}|^{2}+\mathscr{C}_{0}(\varepsilon(\boldsymbol{u})+\Psi(\nabla u)) \cdot(\varepsilon(\boldsymbol{u})+\Psi(\nabla u))+A_{0}(u, u)\right)\right) \\
& \left.+A_{1}(\dot{u}, \dot{u})+\mathscr{C}_{1}\left(\varepsilon(\dot{\boldsymbol{u}})+\partial_{t} \Psi(\nabla u)\right) \cdot\left(\varepsilon(\dot{\boldsymbol{u}})+\partial_{t} \Psi(\nabla u)\right]+\partial_{t} P_{k}(u+g)\right) d x d t \\
& +\int_{S} \partial_{t} \tilde{Q}_{k}\left(\widetilde{u}_{n}\right) d x_{s} d t=\int_{Q_{s}}(\boldsymbol{F} \cdot \dot{\boldsymbol{u}}+f \dot{w}) d x d t \cdot+R\left(u^{(0)}\right),
\end{aligned}
$$


where $\tilde{Q}: r \mapsto \int_{\infty}^{r} q(\zeta) d \zeta$ and $R\left(u^{(0)}\right)$ sums up all the terms containing $u^{(0)}$ or its derivatives. Using the coercivity of the form $A_{i}$ and the form of the operators $\mathscr{C}_{i}$ we obtain the estimate

$$
\begin{aligned}
& \|\dot{u}(s)\|_{H^{1}(\Omega)}^{2}+\|u(s)\|_{H^{2}(\Omega)}^{2}+\|\dot{\boldsymbol{u}}(s)\|_{\boldsymbol{L}_{2}(\Omega)}^{2}+\|\varepsilon(\boldsymbol{u})(s)+\Psi(\nabla u)(s)\|_{L_{2}(\Omega ; \mathbb{S})}^{2} \\
& +\|\dot{u}\|_{L_{2}\left(I_{s} ; H^{2}(\Omega)\right)}^{2}+\left\|\varepsilon(\dot{\boldsymbol{u}})+\partial_{t} \Psi(\nabla u)\right\|_{L_{2}\left(Q_{s} ; \mathbb{S}\right)}^{2}+\left\|P_{k}(u(s)+g)\right\|_{L_{1}(Q)}+ \\
& \left\|\tilde{Q}_{k}\left(\widetilde{u}_{n}(s)\right)\right\|_{L_{1}(S)} \leq C\left(\boldsymbol{u}^{(0)}, \boldsymbol{u}^{(1)}, u^{(0)}, u^{(1)}, \boldsymbol{F}, f\right) \forall s \in(0, T] .
\end{aligned}
$$

Applying the continuous imbedding $H^{2}(\Omega) \hookrightarrow W_{4}^{1}(\Omega)$ we obtain the estimate

$$
\|\Psi(\nabla u)(s)\|_{L_{2}(\Omega ; \mathbb{S})}+\left\|\partial_{t} \Psi(\nabla u)\right\|_{L_{2}\left(I_{s} ; L_{2}(\Omega ; \mathbb{S})\right)} \leq C\left(\boldsymbol{u}^{(0)}, \boldsymbol{u}^{(1)}, u^{(0)}, u^{(1)}, \boldsymbol{F}, f\right) \forall s \in(0, T]
$$

which implies

$$
\|\varepsilon(\boldsymbol{u})(s)\|_{L_{2}(\Omega ; \mathbb{S})}+\|\dot{\varepsilon}(\boldsymbol{u})\|_{L_{2}\left(I_{s} ; L_{2}(\Omega ; \mathbb{S})\right)} \leq C\left(\boldsymbol{u}^{(0)}, \boldsymbol{u}^{(1)}, u^{(0)}, u^{(1)}, \boldsymbol{F}, f\right) \forall s \in(0, T] .
$$

Using the coerciveness of strains (see e.g. [6], Thm 1.2.3) we obtain

$$
\left.\|\boldsymbol{u}(s)\|_{\boldsymbol{H}^{1}(\Omega)}+\|\dot{\boldsymbol{u}}\|_{L_{2}\left(I_{s} ; \boldsymbol{H}^{1}(\Omega)\right)} \leq C\left(\boldsymbol{u}^{(0)}, \boldsymbol{u}^{(1)}, u^{(0)}, u^{(1)}, \boldsymbol{F}, f\right)\right) \forall s \in(0, T]
$$

which together with (46) implies the a priori estimate

$$
\begin{aligned}
& \|\dot{\boldsymbol{u}}\|_{L_{\infty}\left(I ; \boldsymbol{L}_{2}(\Omega)\right)}+\|\dot{\boldsymbol{u}}\|_{L_{2}\left(I ; \boldsymbol{H}^{1}(\Omega)\right)}+\|\boldsymbol{u}\|_{L_{\infty}\left(I ; \boldsymbol{H}^{1}(\Omega)\right)}+\|\dot{u}\|_{L_{\infty}\left(I ; H^{2}(\Omega)\right)}+\|\dot{u}\|_{L_{2}\left(I ; H^{2}(\Omega)\right)} \\
& +\|u\|_{L_{\infty}\left(I ; H^{2}(\Omega)\right)}+\left\|P_{k}(u+g)\right\|_{L_{\infty}\left(I ; L_{1}(Q)\right)}+\left\|\tilde{Q}_{k}\left(\widetilde{u}_{n}\right)\right\|_{L_{\infty}\left(I ; L_{1}(S)\right]} \leq \\
& C\left(\boldsymbol{u}^{(0)}, \boldsymbol{u}^{(1)}, u^{(0)}, u^{(1)}, \boldsymbol{F}, f\right)
\end{aligned}
$$

Since there is no substantial difference between the proof of the solvability of our approximate problem and that of the penalized problem treated in [3], we postpone the readers to that paper for details. Via the standard method it is proved that such a solution is unique.

As in all previous problems the main task is to perform the limit process $k \rightarrow \infty$ for which the $k$-independent estimates of the acceleration terms are needed. To estimate $\ddot{\boldsymbol{u}}_{k} \in L_{2}\left(I ; \boldsymbol{H}^{-1}(\Omega)\right)$ we put an arbitrary $\boldsymbol{w} \in L_{2}\left(I ; \stackrel{\circ}{H}^{1}(\Omega)\right)$ in the approximate variant of (43) and use (47)). To get the estimate $\ddot{u} \in L_{1}\left(I ; H^{2}(\Omega)^{*}\right)$ we must assume (3) which yields the uniform estimate for $\left\|p_{k}\left(u_{k}+g\right)\right\|_{L_{1}(Q)}$ as in Sec. 2. Then we are in the same situation as in Example 3, the Aubin Lemma gives us the crucial strong $L_{2}(Q)$-convergence of all components of velocities. As earlier this leads to the upper semicontinuity of $\left\langle p_{k}\left(u_{k}\right) u_{k}\right\rangle_{Q}$ and $\left\langle\tilde{q}_{k}\left(\widetilde{\left(u_{k}\right)_{n}}\right) \widetilde{\left(u_{k}\right)_{n}}\right\rangle_{S}$ and finally to the fact that $p_{k}\left(u_{k}+g\right) \rightarrow p(u+g)$ in $\mathscr{X}_{1}^{*}$ as in Sec. 2 and $\tilde{q}_{k}\left(\widetilde{\left(u_{k}\right)_{n}}\right) \rightarrow \tilde{q}\left(\widetilde{u_{n}}\right)$ in $L_{\infty}^{*}(S)$ and we are done. The existence of solutions to (43) is proved.

Similarly to the previous sections we can formulate the full von Kármán system with the singular memory replacing all "short memory" terms in (39) and (43) by the corresponding singular memory ones. As in the previous cases under the assumption (16) it is possible to pass from the appropriate approximate problem to the original one in such a way that the crucial strong convergence of velocities holds which leads to the same conclusion as mentioned in the previous paragraph. We allow ourselves to leave this case to kind readers as an exercise.

\section{Relation to the Signorini contact}

In this section we shall prove that for a sequence of the problems with the thickness of the interpenetration

$$
\gamma_{\ell} \nearrow 0, \ell \in \mathbb{N}
$$


there is a subsequence of their solutions called $u_{\ell}$ tending to a limit $u$ which is a solution of a problem without interpenetration, i.e. of the appropriate Signorini version of the problem. Since there is the well-known generic nonuniqueness of the solutions to the dynamic contact of the Signorini type related with the lack of information about the amount of the energy conservation in the contact and thus about the development of the solution after the contact, probably nothing more can be proved in general.

The common feature of the problems treated in the previous sections is that the estimates performed there as $k$-independent are also $\gamma$ independent. Hence if we have a sequence $u_{\ell}$ tending weakly or weakly* to $u$ in the spaces for which the a priori and dual estimates have been derived, we have the strong $L_{2}$ convergence $\dot{u}_{\ell} \rightarrow \dot{u}$. Obviously for the full von Kámán system $\dot{\boldsymbol{u}}_{\ell} \rightarrow \dot{\boldsymbol{u}}$ in $\boldsymbol{L}_{2}(Q)$ holds as well (cf. [7]). Since $u_{\ell}+g \geq \gamma_{\ell}$ a.e. in $Q$, we have $u+g \geq 0$ a.e. there. Moreover for the full von Kármán system we get similarly $\widetilde{u_{n}} \leq 0$. We define

$$
\begin{aligned}
& \mathscr{K} \equiv\left\{v \in \mathscr{X}_{0} ; v \geq-g \text { a.e. in } Q\right\} \text { for problems in Sections } 2,3 \\
& \mathscr{K} \equiv\left\{\{v, \boldsymbol{\omega}\} \in\left(u_{0}+\stackrel{\circ}{H}^{1}(Q)\right) \times \boldsymbol{X}(Q) ; v \geq-g \text { a.e. in } Q\right\} \text { for Reissner-Mindlin plates, } \\
& \mathscr{K} \equiv\left\{\{\boldsymbol{w}, v\} \in \boldsymbol{Y}_{0} ; \boldsymbol{w}_{n} \leq 0 \text { a.e. in } S, v \geq-g \text { a.e. in } Q\right\} \text { for full von Kármán system, }
\end{aligned}
$$

if we define $\boldsymbol{X}(Q)=L_{2}(I ; \boldsymbol{X}(\Omega))$, cf. (2), (26), and (44). Obviously in all cases $p(v+g)=0$ if $v$ is (possibly a component) from $\mathscr{K}$ and, moreover, $q\left(w_{n}\right)=0$ in the last case. Denoting $\Theta \equiv \lim _{\ell \rightarrow \infty}\left\langle p_{\ell}\left(u_{\ell}+g\right), u_{\ell}\right\rangle_{Q}$ and $\vartheta \equiv \lim _{\ell \rightarrow \infty} p_{\ell}\left(u_{\ell}+g\right)$, the monotonicity of $p_{\ell}$ used for the couple $\left\{u_{\ell}, u\right\}$ yields $\Theta \geq \vartheta u$. On the other hand, we can derive from (2) (the solution there must be denoted by $u_{\ell}$ ) with $v=u_{\ell}-y, y \in \mathscr{K}$ the opposite inequality, because in general for $y \in \mathscr{K}$ the lower semicontinuity of $\mathscr{A}$ and $\mathscr{B}$ in the limit process $\ell \rightarrow \infty$ yields

$$
\begin{aligned}
& -\langle\dot{u}, \dot{y}-\dot{u}\rangle_{Q}+\langle\mathscr{A} u, y-u\rangle_{Q}+\langle\mathscr{B} u, y-u\rangle_{Q}+\langle\mathscr{E} u, y-u\rangle_{Q}+\langle\vartheta, y\rangle_{Q}-\Theta \\
& +\langle\dot{u}(T, \cdot),(y-u)(T, \cdot)\rangle_{\Omega} \geq\langle f, y-u\rangle_{Q}+\left\langle u_{1}, y(0, \cdot)-u_{1}\right\rangle_{\Omega}
\end{aligned}
$$

and putting $y=u$ we are done. Hence (50) holds just without the terms with $\vartheta$ and $\Theta$ and this is the exact formulation of the corresponding Signorini problem with $u$ being its solution. This pattern can be exactly followed also in all other cases, because their variational formulations contain only some lower semicontinuous parts, strongly converging terms and linear terms for which the weak convergence is sufficient, hence we prove everytimes $\Theta=\langle\vartheta, u\rangle_{Q}$ and then we can see that the resulting limit variational inequality is the variational formulation of the corresponding Signorini problem indeed. Of course, for Reissner-Mindlin plates we keep the second equation of (26) in the original form observing that the convergences which remain weak there are sufficient.

We only mention the full von Kármán system more in detail. We denote

$$
\Lambda \equiv \lim _{\ell \rightarrow \infty}\left\langle q\left(\widetilde{\left(u_{\ell}\right)_{n}}\right), \widetilde{\left(u_{\ell}\right)_{n}}\right\rangle_{S} \text { and } \lambda \equiv \lim _{\ell \rightarrow \infty} q\left(\widetilde{\left(u_{\ell}\right)_{n}}\right)
$$

and derive immediately that $\Lambda \geq\left\langle\lambda, \widetilde{u_{n}}\right\rangle_{S}$. Denoting the solution of (43) by $\left\{\boldsymbol{u}_{\ell}, u_{\ell}\right\}$ we put $\left\{\boldsymbol{v}-\boldsymbol{u}_{\ell}, w-u_{\ell}\right\}$ as a test function in (43) for an arbitrary $\{\boldsymbol{v}, w\} \in \mathscr{K}$. Then we perform the limit process $\ell \rightarrow \infty$, denote by $\{\boldsymbol{u}, u\}$ the limit of $\left\{\boldsymbol{u}_{\ell}, u_{\ell}\right\}$ and put $\{\boldsymbol{w}, v\}=\{\boldsymbol{u}, u\}$ as a test function into the resulting inequality. Thus we find $\Lambda=\left\langle\lambda, \widetilde{u_{n}}\right\rangle_{S}$ from the first row of it while $\Theta=\langle\vartheta, u\rangle_{Q}$. From this we get that the resulting inequality is in fact the variational formulation of the Signorini contact for the full von Kármán system and $\{\boldsymbol{u}, u\}$ is its solution.

\section{Conclusion}

Existence of solutions has been proved for the dynamic contact problems with limited interpenetration for viscoelastic variants of several classical models of plates. These results are 
now available for technical practice. Probably the most challenging task for the application is the determination of the function $p$ which describes the interpenetration. Performing some sensitivity analysis with respect of its choice may help here.

\section{References}

[1] I. Bock and J. Jarušek. Unilateral dynamic contact of viscoelastic von Kármán plates Adv. Math. Sci. Appl. 16 (1) (2006), 175-187.

[2] I. Bock and J. Jarušek, Unilateral dynamic contact of von Kármán plates with singular memory. Appl. Math. $\mathbf{5 2}(6)(2007), 515-527$.

[3] I. Bock and J. Jarušek. Dynamic contact problem for a bridge modeled by a viscoelastic full von Kármán system. Z. Angew. Math. Phys. 61 (5) (2010), 865-876.

[4] I. Bock and J. Jarušek. Unilateral dynamic contact problem for viscoelastic Reissner-Mindlin plates. Nonlin. Anal., Theory Meth. Appl. 74 (12), (2011), 4192-4202.

[5] J.M. Borwein, J and Q.J. Zhu. Techniques of Variational Analysis. Springer, New York: 2005.

[6] C. Eck, J. Jarušek and M. Krbec: Unilateral Contact Problems: Variational Methods and Existence Theorems, Monographs and Textbooks in Pure and Applied Mathematics 270, Chapman/CRC Press, Providence R.I. etc., 2005.

[7] C. Eck, J. Jarušek and J. Stará. Normal Compliance Contact Models with Finite Interpenetration. Arch. Ration. Mech. Anal. 208 (1) (2013), 25-57.

[8] J. Jarušek. Static semicoercive normal compliance contact problem with limited interpenetration. Zeitschr. Angew. Math. Phys. 66 (20150, 2161-2172.

[9] J. Jarušek and J. Stará. Solvability of a rational contact model with limited interpenetration in viscoelastodynamics. Math. Mech. Solids 23 (7) (2018), 1040-1048.

[10] H. Koch and A. Stachel. Global existence of classical solutions to the dynamic von Kármán equations. Math. Meth. Appl. Sci. 16 (1993), 581-586.

[11] J.E. Lagnese. Boundary Stabilization of Thin Plates. SIAM, Philadelphia, PA, 1989.

[12] A. Signorini. Sopra alcune questioni di statica dei sistemi continui. Ann Scuola Norm-Sup. 2 (1933): 231251.

[13] A. Signorini. Questioni di elasticità non linearizzata e semilinearizzata, Rend. Mat. 18 (1959), 95-139. 